\title{
ВЗАИМОСВЯЗЬ ЛИЧНОСТИ И КРАТКОВРЕМЕННОЙ ПАМЯТИ: РОЛЬ ЧЕРТ И РЕФЛЕКСИВНЫХ АДАПТАЦИЙ ХАРАКТЕРА
}

\section{С.А. ЩЕБЕТЕНКО}

${ }^{a}$ Федеральное государственное бюджетное образовательное учреждение высшего профессионального образования «Пермский государственный национальный исследовательский университет», 614990, Россия, Пермь, ул. Букирева, д. 15

\section{Резюме}

Хотя взаимосвязь познавательной и личностно-мотивационной сфер традиционно привлекает внимание исследователей, анализ литературы показывает, что связи черт личности с кратковременной памятью уделяется крайне мало внимания - в сравнении с исследованиями роли центральных исполнительных структур рабочей памяти (Baddeley, 2003). Кроме того, в этих исследованиях речь преимущественно идет об отдельных чертах личности или о традиционных личностных таксономиях, но не об их производных. В этой связи в работе рассматривается роль рефлексивных адаптаций характера как структурного элемента системы личности в терминах пятифакторной теории (McCrae, Costa, 1996, 2013). На выборке в 1030 человек было установлено, что пространственная кратковременная память, измеренная тестом Корси, коррелировала с нейротизмом и низкой открытостью опыту. Напротив, вербальная кратковременная память, измеренная задачей Стернберга, коррелировала с низкими значениями добросовестности и доброжелательности. Таким образом, в отличие от центрального исполнителя рабочей памяти увеличение значений ее подчиненных систем - пространственной и вербальной кратковременной памяти - сопровождалось низкими значениями доброжелательности, добросовестности и открытости опыту. Кроме того, кратковременная память демонстрировала прямые, опосредованные чертами личности и компенсаторные (в сравнении с чертами) связи с рефлексивными адаптациями характера. В частности, хотя нейротизм не коррелировал с вербальной кратковременной памятью, с последней была связана отрицательная установка на эту черту личности. Аналогично, хотя доброжелательность не коррелировала с пространственной кратковременной памятью, с ней коррелировало отрицательное отношение к доброжелательности, приписываемое родителям (отраженная установка на доброжелательность). Отрицательная связь добросовестности с вербальной кратковременной памятью дополнялась положительной отраженной установкой на эту черту личности. В статье осуществляется анализ результатов в сопоставлении с результатами ранее проведенных исследований и определяются дальнейшие перспективы.

Ключевые слова: пятифакторная теория личности. черты личности, адаптации характера, кратковременная память, рабочая память. 
Индивидуальные различия в поведении могут характеризоваться не только личностными и темпераментальными особенностями, но и вариативностью в познавательной сфере. В частности, кратковременная память может демонстрировать разную эффективность у разных людей (Gathercole, 1999), и потенциально эта вариативность может быть сопряжена с вариативностью в чертах личности. Несмотря на очевидность проблемы, обзор литературы показал, что крайне мало исследований посвящено связи личности с подчиненными системами (slave systems) рабочей памяти (Baddeley, 2003; Baddeley, Hitch, 1974) - фонологической петлей и зрительно-пространственным наброском, - а также со сближающимися с ними в трактовке видами кратковременной памяти $^{1}$ - вербальной и пространственной. Более того, под личностными структурами в существующих исследованиях понимаются обычно черты или свойства личности и не рассматривается роль иных производных структуры личности. Изучению этих проблем посвящена данная работа.

\section{Черты личности, рабочая и кратковременная память}

Способность когнитивной системы к первичной обработке стимулов, поступающих из внешней среды, к хранению и манипулированию релевантной им информацией ограниченного объема в короткие промежутки времени (до 30 с) называется кратковременной памятью (КП) (Заика, Кузнецов, 1989; Солсо, 2006). Хранение и обработка информации в таком режиме являются достаточно затратной функцией с точки зрения ресурсов когнитивной системы, предполагающей большой процент брака, особенно при высокой когнитивной нагрузке (Gathercole, 1999). Индивидуальная вариативность КП может быть связана с самыми различными факторами - от возраста индивида (Там же) и нейронной активности в передней поясной коре (Gray, Braver, 2002) до малоимпульсивного стиля принятия решений (Hinson et al., 2003). Вносят ли черты личности вклад в вариативность КП, и если да, то какой?

Обзор литературы показал, что в подавляющем большинстве случаев исследователей интересует вопрос о связи черт личности с исполнительными функциями, или с так называемым центральным исполнителем (central executive) рабочей памяти (Baddeley, 2003; Baddeley, Hitch, 1974). Так, отдельные авторы предполагают, что импульсивность как черта личности и когнитивные исполнительные функции образуют два полюса единого конструкта

${ }^{1}$ Далее в статье преимущественно используются понятия пространственной и вербальной кратковременной памяти, но предполагается их идентичность с указанными выше подчиненными системами рабочей памяти. В литературе термин «рабочая (оперативная) память» может использоваться как синоним КП (например: Аведисова и др., 2004), либо, в сравнении с КП, как более инклюзивное, комплексное явление (Gathercole, 1999; Nairne, 1996), либо как явление более высокого порядка с точки зрения управления обработкой информации (Baddeley, 2003). 
(Bickel et al., 2012). Действительно, ранее были получены данные об отрицательной связи исполнительных функций с нейротизмом (Williams et al., 2010). При этом дифференциальный анализ исполнительных функций показал, что нейротизм связан с корректировкой/ мониторингом в рабочей памяти (Linnenbrink et al., 1999; Murdock et al., 2013), когнитивной гибкостью (Compton, 2000) и торможением нерелевантной информации (Muris et al., 2009).

В литературе периодически сообщается о связях и других черт личности с исполнительным компонентом рабочей памяти. В частности, экстраверсия положительно коррелировала с корректировкой/мониторингом (Campbell et al., 2011; Lieberman, Rosenthal, 2001; Murdock et al., 2013), а открытость опыту - c когнитивной гибкостью и корректировкой/мониторингом (DeYoung et al., 2005; Murdock et al., 2013; Savine et al., 2012). Доброжелательность была положительно связана с когнитивной гибкостью и торможением нерелевантной информации (JensenCampbell et al., 2002), а добросовестность - с корректировкой/мониторингом (Fleming et al., 2015; JensenCampbell et al., 2002). В то же время H. Ансворт с коллегами (Unsworth et al., 2009) представили противоречащие приведенным выше фактам данные об отрицательных, но незначимых корреляциях между рабочей памятью и добросовестностью, $r=-0.16$, доброжелательностью, $r=-0.18$, и экстраверсией, $r=-0.19$.

Таким образом, исследователи обращаются к интуитивно понятной проблеме - связи черт личности с центральным исполнителем. Как же обстоят дела с ролью систем рабочей памяти, которые считают подчиненными, а именно - зрительно-пространственным наброском и фонологической петлей (Baddeley, 2003)? Связаны ли черты личности с пространственной и зрительной КП? Хотя взаимосвязь познавательной и личностно-мотивационной сфер традиционно привлекает внимание исследователей (см., например, обзор: Семяшкин, 2010), анализ литературы показывает, что обсуждаемой здесь проблеме уделяется крайне мало внимания.

Редкое исключение составляют работы, выполненные в рамках теории контроля внимания (attentional control theory - Derakshan, Eysenck, 2009; Eysenck et al., 2005). Надо отметить, что и здесь речь идет не о личностных таксономиях, а о вкладе диспозициональной тревожности (trait anxiety) в рабочую память, в том числе - в ее подчиненные системы. В то же время в отношении прочих параметров личности нам не удалось обнаружить каких-либо релевантных исследований - как в отечественной, так и в зарубежной литературе.

Другой аспект рассматриваемой проблемы связан с трактовкой личности в опубликованных работах. Преимущественно речь в них идет об отдельных чертах, вроде тревожности (Eysenck et al., 2005) и шизотипии (Park, McTigue, 1997), или о традиционных таксономиях, таких как пятифакторная модель (например: Unsworth et al., 2009). Однако можно предположить, что варьирование КП связано не только с конвенциональными чертами, но и их производными, 
существование которых допускает, в частности, пятифакторная теория личности (ПФТ - McCrae, Costa, 1996, 2013). Так, в терминах ПФТ такие производные могут быть отнесены к разряду адаптаций характера.

\section{Рефлексивные адаптации характера}

ПФТ предполагает, что структуры личности могут быть дифференцированы на два больших подкласса: базовые тенденции (basic tendencies) и характерные адаптации, или адаптации характера (characteristic adaptations - McCrae, Costa, 1996, 2013). «Большая пятерка» черт личности относится к базовым тенденциям и в этом смысле имеет прямую биологическую детерминацию, содержательно сближаясь с понятием темперамента (McCrae, Costa, 2013, p. 18). Напротив, адаптации характера обладают преимущественно социокультурным происхождением и включают в себя потенциально широкий (но не очерченный теоретически) круг личностных феноменов. Среди них можно выделить отдельный подкласс, связанный со способностью индивида рефлексировать черты личности. Этот подкласс можно назвать рефлексивными адаптациями характера, которые дифференцируются как минимум на четыре вида (Щебетенко, 2015б): диспозициональную эффективность, установки на черты, отраженные черты и отраженные установки на черты. Диспозициональная эффективность определяется как суждения индивида о своей эффективности в ситуациях, стимулирующих проявление данной черты. Установка на черту понимается как валентная (положительная/отрицательная) оценка индивидом данной черты без прямого ее отнесения к собственной личности (Shchebetenko, 2014). Отраженная черта понимается как мнение индивида о том, как его черта воспринимается значимыми другими. Наконец, отраженная установка на черту трактуется как мнение индивида о том, какими установками на данную черту обладают значимые другие (Щебетенко, 2015а).

Рефлексивные адаптации характера могут вносить уникальный вклад в объяснение взаимосвязей черт личности с такими внешними критериями, как восприятие живописи (Щебетенко, Тютикова, 2015), успеваемость (Щебетенко, 2015а) и социометрический статус (Балабина, 2015). В этой связи можно предположить, что рефлексивные адаптации характера продемонстрируют связи с КП, независимые или инкрементные в отношении черт личности.

Структурная конфигурация рассматриваемых в данном исследовании переменных определяется следующими соображениями. Теоретически в терминах пятифакторной модели КП не является внешним продуктом ключевых элементов системы личности, так называемой объективной биографией (objective biography). Напротив, КП скорее можно интерпретировать как базовую тенденцию, альтернативную чертам личности. Р. Мак-Крей и П. Коста (McCrae, Costa, 2013) отмечают, что базовые тенденции не сводятся исключительно к «большой пятерке» черт. Напротив, базовые тенденции могут включать в себя и другие индивидуальные параметры, имеющие 
ярко выраженную биологическую детерминацию. Предположительно таковыми могут быть и мнестические процессы вроде КП (например: Bishop et al., 2006). В таком случае следует предположить, что варьирование КП может влиять на адаптации характера, в том числе рефлексивные. Черты личности как базовые тенденции потенциально могут опосредовать эти эффекты в той мере, в которой КП может быть частной причиной варьирования (или ковариатой) черт личности.

\section{Пространственная и вербальная кратковременная память}

Согласно классической версии теории А. Бэддли (Baddeley, 2003), структура рабочей памяти включает в себя две подчиненные системы зрительно-пространственный набросок (visuospatial sketchpad) и фонологическую петлю (phonological loop), соответствующие пространственной и вербальной КП, и могут иметь различные нейрогенетические основания (Baddeley, Hitch, 1974; Smith, Jonides, 1997; Wang, Bellugi, 1994). Эти основания, в частности, выражаются в межполушарной асимметрии (Gathercole, 1999) и в дифференциации симптомов при генетических патологиях (Jarrold, Baddeley, 1997). В данном исследовании для измерения пространственной и вербальной КП использовались тест П. Корси (Corsi, 1972) и задача С. Стернберга (Sternberg, 1966) соответственно. В терминах модели А. Бэддли и Г. Хитча (Baddeley, Hitch, 1974) результаты теста Корси адекватны зрительно-про- странственному наброску (McLean, Hitch, 1999), а задачи Стернберга фонологической петле (Gathercole, 1999). Ранее тест Корси использовался при измерении КП (FeriniStrambi et al., 2003; Feuerstein et al., 1997), рабочей памяти (Naegele et al., 1998), мнемической эффективности (Feuerstein et al., 1997). Тест Корси чувствителен к кратковременной памяти индивида с минимальным обращением к его долговременной памяти (Bull et al., 2008).

Классическая парадигма (задача) C. Стернберга (Sternberg, 1966) использовалась для изучения времени воспроизведения вербальной информации из КП. Основной результат парадигмы заключается в том, что среднее время реакции линейно возрастает в связи с числом пунктов в серии предъявляемых вербальных стимулов (букв или цифр).

Целью данного исследования было изучение того, как связаны между собой КП и личностные структуры, определенные в ПФТ. Поскольку для постановки эксплицитных гипотез, судя по обзору литературы, недостаточно фактологического и теоретического материала, я ограничился формулированием двух исследовательских вопросов.

Q1: Демонстрируют ли пространственная и вербальная разновидности кратковременной памяти различные паттерны связи с чертами личности?

Q2: Оказывает ли кратковременная память прямые воздействия на рефлексивные адаптации характера или они опосредованы чертами личности как базовыми тенденциями? 


\section{Метод}

\section{Участники исследования}

В исследовании приняли участие 1030 студентов одного из российских университетов в возрасте от 17 до 38 лет $(\mathrm{M}=19.65, \mathrm{SD}=1.72)$. Одна участница не сообщила своего возраста. Среди участников была 691 женщина (67.1\%).

\section{Измерение}

Личностные характеристики. Участники заполняли русскую версию (Shchebetenko, 2014) 44-пунктного «Вопросника Большой Пятерки» (Big Five Inventory, BFI John et al., 1991, 2008), а также ряд его модификаций, измеряющих рефлексивные адаптации характера. В части отраженных черт и отраженных установок участников просили обратиться к образу их родителей, поскольку последние могут быть эффективной формой значимого другого для студентов (Malloy et al., 1997). Подшкалы всех вопросников продемонстрировали приемлемый уровень внутренней согласованности, $0.66<\alpha<0.87$. Исключение составили подшкалы «Установка на экстраверсию» и «Отраженная установки на экстраверсию», $\alpha=0.57$ и 0.51 соответственно. Для увеличения надежности из этих подшкал были исключены три пункта: «сдержанность», «порождение энтузиазма» и «застенчивость», в результате чего внутренняя согласованность увеличилась до $\alpha=0.69$ и 0.65 соответственно.

Кратковременная память. Участники индивидуально выполняли тест Корси и задачу Стернберга в лаборатории, работая в группах от 3 до 15 человек. Использовались скрипты этих тестов для программы Inquisit 3.0 компании Millisecond (Inquisit 3.0, 2015). Инструкции тестов предварительно переводились на русский язык.

В тесте Корси участникам предъявлялись девять квадратов, размещенных в произвольном порядке на плоскости (экране). Квадраты в случайной последовательности меняли свой цвет (с синего на желтый и обратно), образуя серию. После этого участник должен был воспроизвести эту серию посредством курсора и мышки. Размер серии постепенно увеличивался: задание начиналось с серии в два квадрата и продолжалось до тех пор, пока участник не совершал ошибки в двух последовательных попытках для серии одинаковой длины или не достигал предельного максимума в девять квадратов. В таком виде тест Корси измеряет пространственную КП, показателем которой является количество баллов, рассчитываемых от количества успешно выполненных серий ${ }^{2}$.

В задаче Стернберга участники изучали список из временно́й серии вербальных пунктов (цифры), после

\footnotetext{
${ }^{2}$ Возможен также реверсивный порядок, валидный замеру комплексной рабочей памяти: в этом случае участник должен воспроизвести серию в порядке, обратном тому, который предъявил экспериментатор, т.е. активно обработать информацию из пространственной КП (Bull et al., 2008). Этот показатель в настоящем исследовании не измерялся.
} 
которого следовал тестовый стимул. Участник должен был как можно быстрее определить, был ли тестовый стимул представлен в изначальной временно́й серии. Задание повторялось 18 раз, а длина каждого стимульного блока случайно варьировалась от двух до семи элементов: таким образом, каждый участник выполнял по три попытки для серий каждого из шести возможных размеров. В качестве показателя, релевантного вербальной КП, использовалось обратное число допущенных ошибок при воспроизведении стимула.

Данные пяти участников по тесту Корси и двух участников по задаче Стернберга были утеряны. По всей видимости, это произошло случайно относительно значений их КП в генеральной совокупности, и, соответственно, отсутствующие значения заменялись методом линейного тренда к точке.

Показатель пространственной КП имел умеренную положительную асимметрию, g1 $=0.87$, и существенный положительный эксцесс, g2 = 1.22. Показатель вербальной КП имел существенные отрицательную асимметрию, g1 = -2.38, и положительный эксцесс, g2 = 5.43. Для сокращения отрицательной асимметрии показатель вербальной КП возводился в куб (Revelle, 2012), что привело к значительному сокращению асимметрии и эксцесса, g1 = -1.31 и g2 = 1.42. Однако поскольку эти значения тем не менее выходят за пределы рекомендуемых, в дополнение для оценки статистической достоверности использовалась робастная статистика бутстраппинг. Ранее было показано, что бутстраппинг является эффективным сред- ством контроля ошибки I рода при нарушениях нормальности распределения (Berkovits et al., 2000; Wilcox et al., 2013).

\section{Результаты}

\section{Кратковременная память: \\ дифферениированныцй паттерн корреляций со структурами личности}

Пространственная КП слабо и отрицательно коррелировала с нейротизмом и открытостью опыту (таблица 1). Вербальная КП отрицательно коррелировала с добросовестностью и доброжелательностью. Кроме того, пространственная КП отрицательно коррелировала с диспозициональной эффективностью в нейротизме, отраженным нейротизмом и отраженной установкой на доброжелательность. Вербальная КП отрицательно коррелировала с диспозициональной эффективностью в добросовестности, отраженными добросовестностью и доброжелательностью, а также установкой на нейротизм. Кроме того, корреляции вербальной КП с двумя отраженными установками приблизились к конвенциональным уровням значимости. При этом с отраженной установкой на добросовестность вербальная КП коррелировала положительно, а с отраженной установкой на нейротизм - отрицательно.

\section{Кратковременная память и рефлексивные адаптащии характера: прямые эффекты и опосредования чертами личности}

Пространственная КП. Черта нейротизма опосредовала связь про- 
Таблица 1

Корреляции показателей кратковременной памяти с характеристиками личности

\begin{tabular}{|c|c|c|}
\hline \multirow{2}{*}{ Характеристики личности } & \multicolumn{2}{|c|}{ Кратковременная память } \\
\hline & Пространственная (Корси) & Вербальная (Стернберг) \\
\hline \multicolumn{3}{|c|}{ Черты личности } \\
\hline Экстраверсия & $-0.03[-0.09 ; 0.03]$ & $-0.01[-0.06 ; 0.05]$ \\
\hline Доброжелательность & $0.01[-0.05 ; 0.07]$ & $-0.07[-0.13 ;-0.02]$ \\
\hline Добросовестность & $-0.03[-0.10 ; 0.04]$ & $-0.11[-0.16 ;-0.05]$ \\
\hline Нейротизм & $-0.09[-0.16 ;-0.03]$ & $-0.01[-0.07 ; 0.04]$ \\
\hline Открытость опыту & $-0.07[-0.13 ;-0.01]$ & $0.02[-0.04 ; 0.08]$ \\
\hline \multicolumn{3}{|c|}{ Установки на черты } \\
\hline Экстраверсия & $-0.01[-0.07 ; 0.05]$ & $-0.05[-0.10 ; 0.01]$ \\
\hline Доброжелательность & $-0.02[-0.08 ; 0.03]$ & $-0.05[-0.10 ; 0.01]$ \\
\hline Добросовестность & $0.01[-0.05 ; 0.07]$ & $-0.04[-0.10 ; 0.03]$ \\
\hline Нейротизм & $-0.04[-0.09 ; 0.02]$ & $-0.11[-0.18 ;-0.05]$ \\
\hline Открытость опыту & $-0.04[-0.09 ; 0.02]$ & $0.02[-0.04 ; 0.08]$ \\
\hline \multicolumn{3}{|c|}{ Диспозициональная эффективность } \\
\hline Экстраверсия & $-0.01[-0.08 ; 0.05]$ & $-0.03[-0.09 ; 0.03]$ \\
\hline Доброжелательность & $-0.01[-0.07 ; 0.05]$ & $-0.04[-0.10 ; 0.02]$ \\
\hline Добросовестность & $0.00[-0.06 ; 0.06]$ & $-0.08[-0.14 ;-0.02]$ \\
\hline Нейротизм & $-0.12[-0.18 ;-0.06]$ & $0.02[-0.04 ; 0.08]$ \\
\hline Открытость опыту & $-0.03[-0.09 ; 0.03]$ & $0.03[-0.03 ; 0.09]$ \\
\hline \multicolumn{3}{|c|}{ Отраженные черты } \\
\hline Экстраверсия & $-0.04[-0.10 ; 0.02]$ & $-0.04[-0.10 ; 0.02]$ \\
\hline Доброжелательность & $0.03[-0.02 ; 0.09]$ & $-0.08[-0.13 ;-0.02]$ \\
\hline Добросовестность & $-0.04[-0.10 ; 0.02]$ & $-0.11[-0.17 ;-0.05]$ \\
\hline Нейротизм & $-0.09[-0.15 ;-0.03]$ & $-0.01[-0.07 ; 0.05]$ \\
\hline Открытость опыту & $-0.06[-0.12 ; 0.01]$ & $0.04[-0.02 ; 0.10]$ \\
\hline \multicolumn{3}{|c|}{ Отраженные установки на черты } \\
\hline Экстраверсия & $0.03[-0.04 ; 0.09]$ & $0.01[-0.06 ; 0.07]$ \\
\hline Доброжелательность & $-0.07[-0.13 ;-0.01]$ & $0.01[-0.05 ; 0.07]$ \\
\hline Добросовестность & $-0.01[-0.06 ; 0.05]$ & $0.06[0.00 ; 0.14]$ \\
\hline Нейротизм & $0.00[-0.06 ; 0.06]$ & $-0.06[-0.12 ; 0.00]$ \\
\hline Открытость опыту & $-0.05[-0.11 ; 0.01]$ & $-0.01[-0.07 ; 0.05]$ \\
\hline
\end{tabular}

Примечание. Показатель вербальной памяти (число ошибок в задаче Стернберга) реверсирован. Полужирным выделены значения, при которых границы доверительных интервалов не включали в себя ноль, что эквивалентно отвержению нулевой гипотезы на уровне $\alpha=0.05$. 
странственной КП с диспозициональной эффективностью в нейротизме, непрямой эффект (IE) [95\%-й доверительный интервал, полученный на 5000 бутстрап-выборок] $=-0.045$ $[-0.076 ;-0.012], Z=2.67, p=0.008$, а также с отраженным нейротизмом, $\mathrm{IE}=-0.046[-0.080 ;-0.013], \mathrm{Z}=2.67$, $p=0.008$.

Вербальная КП. Черта добросовестности опосредовала связь вербальной КП с диспозициональной эффективностью в добросовестности, IE $=-0.027$ [-0.042; -0.012], $\mathrm{Z}=3.49, p=0.001$, а также с отраженной добросовестностью, IE $=-0.033$ [-0.052; -0.015], $\mathrm{Z}=3.49, p=0.001$. Аналогично, черта доброжелательности опосредовала связь вербальной КП с отраженной доброжелательностью, IE $=-0.018[-0.032$; $-0.004], \mathrm{Z}=2.35, p=0.019$.

Поскольку добросовестность и отраженная установка на эту черту коррелировали с вербальной КП с противоположными знаками, мы оценили не медиацию, а совместный вклад вербальной КП и добросовестности в отраженную установку посредством множественного регрессионного анализа, $R^{2}=0.019$, F (2, $1027)=9.85, p<0.001$. Вклад обоих предикторов в отраженную установку на добросовестность оставался существенным и независимым друг от друга: В [95\%-й СI для 5000 бутстрап-выборок] $=0.015$ [0.001; 0.031], $\beta=0.08, p=0.015$, и $\mathrm{B}=0.070$ [0.033; $0.110], \beta=0.12, p<0.001$, для вербальной КП и добросовестности соответственно. Таким образом, более положительная установка на добросовестность может быть как продуктом собственно добросовестности, так и альтернативно увели- ченной вербальной КП; при этом, напомню, сама добросовестность отрицательно коррелировала с вербальной КП.

\section{Обсуждение}

\section{Связь черт личности с кратковременной памятью}

В настоящем исследовании впервые изучается связь черт личности с двумя видами кратковременной памяти (КП): вербальной и пространственной. Предыдущие исследования касались связи черт личности с ключевым элементом рабочей памяти - центральным исполнителем, не уделяя при этом внимания двум подчиненным кратковременным системам - зрительно-пространственному наброску и фонологической петле. В этом плане представленное исследование восполняет эмпирический пробел.

Пространственная и вербальная КП продемонстрировали различный паттерн связи с чертами личности. Тем самым был получен утвердительный ответ на первый исследовательский вопрос: в то время как пространственная КП отрицательно коррелировала с нейротизмом и открытостью опыту, вербальная КП отрицательно коррелировала с доброжелательностью и добросовестностью.

Отрицательная связь пространственной КП с нейротизмом согласуется с существующими теориями и полученными ранее данными (Еуsenck et al., 2005; Unsworth et al., 2009), которые поддерживают предположение о том, что нейротизм и ограничения рабочей памяти могут 
считаться симптомами расстройства личности. Сравнительный анализ нейропсихологических данных показывает, что они отчасти согласуются с полученным результатом. В частности, в то время как нейротизм отрицательно коррелировал с активностью в дорсолатеральной зоне префронтального кортекса и правой прецентральной извилине (DeYoung et al., 2010), пространственная КП положительно коррелировала с активностью в нижней зоне префронтального кортекса и премоторной коре (Gathercole, 1999).

Представленное исследование показывает, что в отличие от центрального исполнителя рабочей памяти увеличение ее подчиненных систем - пространственной и вербальной КП - может сопровождаться низкими значениями в доброжелательности, добросовестности и открытости опыту. Эти данные скорее согласуются с приведенными выше результатами исследования Н. Ансворт с соавт. (Unsworth et al., 2009): как отмечалось, ими были получены отрицательные корреляции рабочей памяти с экстраверсией, добросовестностью и доброжелательностью, которые в силу сравнительно низкой мощности их исследования $(\mathrm{N}=138)$ были, однако, проинтерпретированы как незначимые.

В частности, в данном исследовании добросовестность и доброжелательность отрицательно коррелировали с вербальной КП. Это свидетельствует о том, что добросовестность и доброжелательность могут выполнять компенсаторную функцию при ограничениях в вербальной КП, что согласуется с ранее предоставленными данными об отрицательной связи добросовестности с интеллектом (Allik, Realo, 1997; Moutafi et al., 2004). В плане интерпретации такой связи Дж. Мутафи с коллегами (Moutafi et al., 2004, p. 1015) предполагают, что менее интеллектуальные индивиды становятся более добросовестными, чтобы справляться со своими когнитивными ограничениями, а более интеллектуальные индивиды становятся менее добросовестными, поскольку они могут полагаться при решении когнитивных задач в основном на свой интеллект. Возможно, добросовестность находится в аналогичных отношениях с вербальной КП. Аналогичным образом ограничения вербальной КП могут стимулировать просоциальное поведение, вызываемое доброжелательностью.

Дж. Костантини с соавт. (Costantini et al., 2015) обнаружили отрицательные корреляции одного из аспектов добросовестности - упорядоченности (orderliness facet), измеренной как самоотчетным эксплицитным, так и имплицитным тестами, с рабочей памятью, измеренной Automated Operation Span Task (Unsworth et al., 2005). Специфика этого теста указывает на то, что он валиден фонологической петле. Таким образом, результаты Костантини с соавт. согласуются с данными настоящего исследования. Однако, поскольку в моем случае анализ аспектов добросовестности не осуществлялся, нельзя определить то, насколько ключевую роль в этой корреляции исполняет аспект упорядоченности. Авторы предлагают двоякую интерпретацию полученных корреляций. Во-первых, они 
также видят сходство их результата с данными об отрицательной связи между добросовестностью и интеллектом. Во-вторых, они предполагают, что организованность и упорядоченность среды может снижать потребность индивида в больших объемах рабочей памяти. Тем самым добросовестность выступает в роли своеобразного ингибитора этого когнитивного ресурса.

Мое исследование не подтверждает предыдущих данных о том, что КП связана с повышенной экстраверсией (Lieberman, Rosenthal, 2001) или открытостью опыту (DeYoung et al., 2005). В более широком смысле, не было найдено свидетельств и тому, что КП необходима для обеспечения пластичности (DeYoung, 2006) в системе личности. Был получен скорее опровергающий факт - отрицательная связь пространственной КП с открытостью опыту. Использованная выше компенсаторная логика может пригодиться и в данном случае: ограничения в пространственной КП могут способствовать дополнительным усилиям индивида по изучению окружающего мира и большей любознательности. Гипотетически возможно, что открытость положительно связана с центральным исполнителем рабочей памяти (согласно предположениям и данным К. ДеЯнга - DeYoung, 2006) при ограничениях в подчиненном ее компоненте - пространственной КП.

\section{Роль рефлексивных адаптаций характера}

Результаты исследования показывают, что КП может влиять на рефлексивные адаптации характера либо напрямую, либо опосредованно чертами личности. В плане прямых эффектов было установлено, что хотя нейротизм не коррелировал с вербальной КП, тем не менее с последней была связана отрицательная установка на эту черту личности. Иными словами, ограничения вербальной КП не связаны с нейротизмом, но, вероятно, приводят к положительному отношению к нему. Аналогично, хотя доброжелательность не коррелировала с пространственной КП, с последней коррелировало отрицательное отношение к доброжелательности, приписываемое родителям.

С другой стороны, был получен ряд непрямых эффектов, свидетельствующих о том, что связь рефлексивных адаптаций характера с КП объясняется их связью с соответствующими чертами личности. Так, ограничения в пространственной КП приводили к увеличению диспозициональной эффективности в нейротизме и отраженному нейротизму. Однако эти эффекты не были самостоятельными, а, напротив, опосредованными повышенным нейротизмом как чертой. Иными словами, ограничения в пространственной КП влияли на увеличение нейротизма, а повышенный нейротизм, в свою очередь, влиял на диспозициональную эффективность в нейротизме и отраженный нейротизм. Аналогичная ситуация наблюдалась в части связи вербальной КП с добросовестностью, доброжелательностью и их производными.

Исключением явилась специфическая связь вербальной КП с отраженной установкой на добросовестность. В данном случае негативная 
связь добросовестности с вербальной КП дополнялась положительным отношением к этой черте, приписываемым родителям. Иными словами, высокие значения вербальной КП могут приводить к снижению добросовестности или пониманию, что эта черта является положительной. Правда, это мнение проецируется на значимых других. Этот факт является примером того, что рефлексивные адаптации характера могут не только производить самостоятельные воздействия или опосредовать эффекты черт личности на внешние критерии, но и выполнять компенсаторную функцию в отношении соответствующей черты.

К очевидным ограничениям полученных результатов относится следующее. Во-первых, исследование носило кросс-секционный характер, поэтому суждения о влиянии КП на черты личности и рефлексивные адаптации являются гипотетическими, хотя и обусловленными теоретическими соображениями. Во-вторых, несмотря на внушительный размер, задействованная выборка была студенческой. Тем самым достоверность полученных результатов ограничена возрастными и образовательными факторами. В-третьих, в силу того, что многие результаты противоречат ранее полученным данным, в дальнейших исследованиях стоит включить в расчеты показатель центрального исполнителя - наряду с показателями служебных систем. В-четвертых, судя по полученным коэффициентам корреляции, связи КП с характеристиками личности имеют слабый характер, значительность которых можно зафиксировать только при выборках достаточной мощности, вроде той, которая была использована в данном исследовании. Иными словами, в исследованиях с выборками сравнительно небольшого размера авторы будут склонны принимать нулевую гипотезу, пример чему обсуждался выше. Это обстоятельство, с другой стороны, увеличивает вероятность угрозы ошибки первого рода и, соответственно, повышает необходимость репликации полученных эффектов. В случае дальнейшего подтверждения представленных в этой работе результатов можно заключить, что КП и характеристики личности связаны между собой слабо, но существенно (о роли слабых эффектов в психологии и необходимости их фиксации см., например: Cumming, 2014).

\section{Литература}

Аведисова, А. С., Файзуллоев, А. А., Бугаева, Т. П. (2004). Динамика когнитивных функций у больных с эмоционально-лабильными расстройствами сосудистого генеза при лечении вазобралом. Клиническая фармакология и терапия, 13(2), 1-4.

Балабина А. Д. (2015). Рефлексивные адаптации характера и социометрический статус. Вестник Пермского университета. Философия. Психология. Социология, 2(22), 55-64.

Заика, Е. В., Кузнецов, М. А. (1989). Кратковременная память и усвоение практических умений. Вопросы психологии, 2, 120-123. 
Семяшкин, А. А. (2010). Соотношение когнитивных стилей и индивидуально-психологических особенностей личности (Кандидатская диссертация, Московский государственный гуманитарный университет им. М.А. Шолохова, Москва).

Солсо, Р. (2006). Когнитивная психология. СПб.: Питер.

Щебетенко, С. А. (2015а). Отраженные установки на черты личности как предиктор успеваемости студентов. Психология и психотехника, 1(76), 70-82. doi:10.7256/2070-8955.2015.1.13526

Щебетенко, С. А. (2015б). Рефлексивные адаптации характера в пятифакторной теории личности. Психологический журнал, 36(6), 55-65.

Щебетенко, С. А., Тютикова, Е. А. (2015). «Картина хороша, потому что хороша открытость опыту»: опосредующая роль установок на черты личности в индивидуальных различиях отношения к живописи. Психология. Журнал Высшей школь экономики, 12(4), 122-141.

Allik, J., \& Realo, A. (1997). Intelligence, academic abilities, and personality. Personality and Individual Differences, 23, 809-814. doi:10.1016/S0191-8869(97)00103-7

Baddeley, A. (2003). Working memory: Looking back and looking forward. Nature Reviews Neuroscience, 4, 829-839. doi:10.1038/nrn1201

Baddeley, A. D., \& Hitch, G. (1974). Working memory. In G. H. Bower (Ed.), Psychology of learning and motivation: Advances in research and theory (Vol. 8, pp. 47-89). New York: Academic Press.

Berkovits, I., Hancock, G. R., \& Nevitt, J. (2000). Bootstrap resampling approaches for repeated measure designs: Relative robustness to sphericity and normality violations. Educational and Psychological Measurement, 60, 877-892. doi:10.1177/00131640021970961

Bickel, W. K., Jarmolowicz, D. P., Mueller, E. T., Gatchalian, K. M., \& McClure, S. M. (2012). Are executive function and impulsivity antipodes? A conceptual reconstruction with special reference to addiction. Psychopharmacology, 221, 361-387. doi:10.1007/s00213-012-2689-x

Bishop, D. V. M., Adams, C. V., \& Norbury, C. F. (2006). Distinct genetic influences on grammar and phonological short-term memory deficits: evidence from 6-year-old twins. Genes, Brain and Behavior, 5, 158-169. doi:10.1111/j.1601-183X.2005.00148.x

Bull, R., Espy, K. A., \& Wiebe, S. A. (2008). Short-term memory, working memory, and executive functioning in preschoolers: Longitudinal predictors of mathematical achievement at age 7 years. Developmental Neuropsychology, 33, 205-228. doi:10.1080/87565640801982312

Campbell, A. M., Davalos, D. B., McCabe, D. P., \& Troup, L. J. (2011). Executive functions and extraversion. Personality and Individual Differences, 51, 720-725. doi:10.1016/j.paid.2011.06.018

Compton, R. J. (2000). Ability to disengage attention predicts negative affect. Cognition and Emotion, 14, 401-415. doi:10.1080/026999300378897

Corsi, P. M. (1972). Human memory and the medial temporal region of the brain. Dissertation Abstracts International, 34(02), 891B. (University Microfilms No. AAI05-77717).

Costantini, G., Richetin, J., Borsboom, D., Fried, E. I., Rhemtulla, M., \& Perugini, M. (2015). Development of indirect measures of conscientiousness: Combining a facets approach and network analysis. European Journal of Personality, 29, 548-567. doi:10.1002/per.2014.

Cumming, G. (2014). The new statistics: Why and how. Psychological Science, 25, 7-29. doi:10.1177/0956797613504966

Derakshan, N., \& Eysenck, M. W. (2009). Anxiety, processing efficiency, and cognitive performance. European Psychologist, 14, 168-176. doi:10.1027/1016-9040.14.2.168

DeYoung, C. G. (2006). Higher-order factors of the Big Five in a multi-informant sample. Journal of Personality and Social Psychology, 91, 1138-1151. doi:10.1037/0022-3514.91.6.1138 
DeYoung, C. G., Hirsh, J. B., Shane, M. S., Papademetris, X., Rajeevan, N., \& Gray, J. R. (2010). Testing predictions from personality neuroscience: Brain structure and the Big Five. Psychological Science, 21, 820-828. doi:10.1177/0956797610370159

DeYoung, C. G., Peterson, J. B., \& Higgins, D. M. (2005). Sources of openness/intellect: Cognitive and neuropsychological correlates of the fifth factor of personality. Journal of Personality, 73, 825-858. doi:10.1111/j.1467-6494.2005.00330.x

Eysenck, M., Payne, S., \& Derakshan, N. (2005). Trait anxiety, visuospatial processing, and working memory. Cognition and Emotion, 19, 1214-1228. doi:10.1080/02699930500260245

Ferini-Strambi, L., Baietto, C., Di Gioia, M. R., Castaldi, P., Castronovo, C., Zucconi, M., \& Cappa, S. F. (2003). Cognitive dysfunction in patients with obstructive sleep apnea (OSA): partial reversibility after continuous positive airway pressure (CPAP). Brain Research Bulletin, 61, 87-92. doi:10.1016/S0361-9230(03)00068-6

Feuerstein, C., Naлgele, B., Pepin, J.-L., \& Levy, P. (1997). Frontal lobe-related cognitive functions in patients with Sleep Apnea Syndrome before and after treatment: The relationship between sleep and cognitive functions during wakefulness. Acta Neurologica Belgica, 97, 96-107. Retrieved from http://cat.inist.fr/?aModele $=$ afficheN\&cpsidt $=2714326$

Fleming, K. A., Heintzelman, S. J., \& Bartholow, B. D. (2015). Specifying associations between conscientiousness and executive functioning: Mental set shifting, not prepotent response inhibition or working memory updating. Journal of Personality. Advanced online publication. doi:10.1111/jopy.12163

Gathercole, S. E. (1999). Cognitive approaches to the development of short-term memory. Trends in Cognitive Sciences, 3, 410-419. doi:10.1016/S1364-6613(99)01388-1

Gray, J. R., \& Braver, T. S. (2002). Personality predicts working-memory-related activation in the caudal anterior cingulate cortex. Cognitive, Affective, and Behavioral Neuroscience, 2, 64-75. doi:10.3758/CABN.2.1.64

Hinson, J. M., Jameson, T. L., \& Whitney, P. (2003). Impulsive decision making and working memory. Journal of Experimental Psychology: Learning, Memory, and Cognition, 29, 298-306. doi:10.1037/0278-7393.29.2.298

Inquisit 3.0. (2015). Computer software. Seattle, WA: Millisecond Software.

Jarrold, C., \& Baddeley, A. D. (1997). Short-term memory for verbal and visuospatial information in Down's syndrome. Cognitive Neuropsychiatry, 2, 101-122. doi:10.1080/135468097396351

Jensen-Campbell, L. A., Rosselli, M., Workman, K. A., Santisi, M., Rios, J. D., \& Bojan, D. (2002). Agreeableness, conscientiousness, and effortful control processes. Journal of Research in Personality, 36, 476-489. doi:10.1016/S0092-6566(02)00004-1

John, O. P., Donahue, E. M., \& Kentle, R. L. (1991). The Big Five Inventory - Versions 4a and 54. Berkeley, CA: University of California, Berkeley, Institute of Personality and Social Research.

John, O. P., Naumann, L. P., \& Soto, C. J. (2008). Paradigm shift to the integrative Big Five trait taxonomy: History, measurement, and conceptual issues. In O. P. John, R. W. Robins, \& L. A. Pervin (Eds.), Handbook of personality: Theory and research (pp. 114-158). New York: Guilford Press.

Lieberman, M. D., \& Rosenthal, R. (2001). Why introverts can't always tell who likes them: Multitasking and nonverbal decoding. Journal of Personality and Social Psychology, 80, 294-310. doi:10.1037/0022-3514.80.2.294

Linnenbrink, E. A., Ryan, A. M., \& Pintrich, P. R. (1999). The role of goals and affect in working memory functioning. Learning and Individual Differences, 11, 213-230. doi:10.1016/S10416080(00)80006-0 
Malloy, T. E., Albright, L., Kenny, D. A., Agatstein, F., \& Winquist, L. (1997). Interpersonal perception and metaperception in nonoverlapping social groups. Journal of Personality and Social Psychology, 72, 390-398. doi:10.1037/0022-3514.72.2.390

McCrae, R. R., \& Costa, P. T., Jr. (1996). Toward a new generation of personality theories: Theoretical contexts for the five-factor model. In J. S. Wiggins (Ed.), The Five-factor model of personality: Theoretical perspectives (pp. 51-87). New York: Guilford Press.

McCrae, R. R., \& Costa, P. T., Jr. (2013). Introduction to the empirical and theoretical status of the five-factor model of personality traits. In T. A. Widiger \& P. T. Costa, Jr. (Eds.), Personality disorders and the five-factor model of personality (3rd ed., pp. 15-27). Washington, DC: American Psychological Association.

McLean, J. F., \& Hitch, G. J. (1999). Working memory impairments in children with specific arithmetic learning difficulties. Journal of Experimental Child Psychology, 74, 240-260. doi:10.1006/jecp.1999.2516

Moutafi, J., Furnham, A., \& Paltiel, L. (2004). Why is conscientiousness negatively correlated with intelligence? Personality and Individual Differences, 37, 1013-1022. doi:10.1016/ j.paid.2003.11.010

Murdock, K. W., Oddi, K. B., \& Bridgett, D. J. (2013). Cognitive correlates of personality: Links between executive functioning and the big five personality traits. Journal of Individual Differences, 34, 97-104. doi:10.1027/1614-0001/a000104

Muris, P., Bos, A. E. R., Mayer, B., Verkade, R., Thewissen, V., \& Dell'Avvento, V. (2009). Relations among behavioral inhibition, Big Five personality factors, and anxiety disorder symptoms in nonclinical children. Personality and Individual Differences, 46, 525-529. doi:10.1016/ j.paid.2008.12.003

Naegele, B., Pepin, J. L., Levy, P., Bonnet, C., Pellat, J., \& Feuerstein, C. (1998). Cognitive executive dysfunction in patients with obstructive sleep apnea syndrome (OSAS) after CPAP treatment. Sleep, 21, 392-397.

Nairne, J. S. (1996). Short-term/working memory. In E. L. Bjork \& R. A. Bjork (Eds.), Memory (pp. 101-128). San Diego, CA: Academic Press.

Park, S., \& McTigue, K. (1997). Working memory and the syndromes of schizotypal personality. Schizophrenia Research, 26, 213-220. doi:10.1016/S0920-9964(97)00051-0

Revelle, W. (2012). An introduction to psychometric theory with applications in R. Retrieved from http://www.personality-project.org/

Savine, A. C., McDaniel, M. A., Shelton, J. T., \& Scullin, M. K. (2012). A characterization of individual differences in prospective memory monitoring using the Complex Ongoing Serial Task.Journal of Experimental Psychology: General, 141, 337-362. doi:10.1037/a0025753

Shchebetenko, S. (2014). "The best man in the world": Attitudes toward personality traits. Psychology. Journal of the Higher School of Economics, 11(3), 129-148.

Smith, E. E., \& Jonides, J. (1997). Working memory: A view from neuroimaging. Cognitive Psychology, 33, 5-42. doi:10.1006/cogp.1997.0658

Sternberg, S. (1966). High-speed scanning in human memory. Science, 153(3736), 652-654. doi:10.1126/science.153.3736.652

Unsworth, N., Heitz, R. P., Schrock, J. C., \& Engle, R. W. (2005). An automated version of the operation span task. Behavior Research Methods, 37, 498-505. doi:10.3758/BF03192720

Unsworth, N., Miller, J. D., Lakey, C. E., Young, D. L., Thadeus, J., Keith, W., \& Goodie, A. S. (2009). Exploring the relations among executive functions, fluid intelligence, and personality. Journal of Individual Differences, 30, 194-200. doi:10.1027/1614-0001.30.4.194 
Wang, P. P., \& Bellugi, U. (1994). Evidence from two genetic syndromes for a dissociation between verbal and visual-spatial short-term memory. Journal of Clinical and Experimental Neuropsychology, 16, 317-322. doi:10.1080/01688639408402641

Wilcox, R., Carlson, M., Azen, S., \& Clark, F. (2013). Avoid lost discoveries, because of violations of standard assumptions, by using modern robust statistical methods. Journal of Clinical Epidemiology, 66, 319-329. doi:10.1016/j.jclinepi.2012.09.003

Williams, P. G., Suchy, Y., \& Kraybill, M. L. (2010). Five-Factor Model personality traits and executive functioning among older adults. Journal of Research in Personality, 44, 485-491. doi:10.1016/j.jrp.2010.06.002

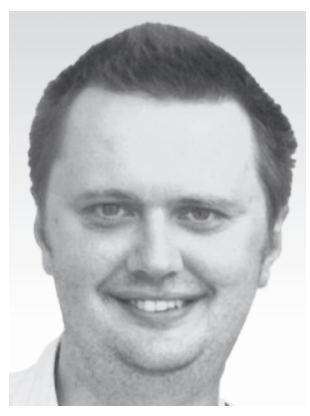

Щебетенко Сергей Александрович - старший научный сотрудник, кафедра психологии развития, Пермский государственный национальный исследовательский университет, кандидат психологических наук, доцент.

Сфера научных интересов: психология личности и индивидуальных различий, стереотипы и предубеждения, имплицитные установки, психометрика, прикладная статистика в психологии. Контакты: shebetenko@rambler.ru

\title{
The Relationship between Personality and Short-Term Memory: The Role of Traits and Reflexive Characteristic Adaptations
}

\author{
Sergei A. Shchebetenko \\ ${ }^{a}$ Perm State University, 15 Bukireva str., Perm, 614990, Russian Federation
}

\begin{abstract}
Although investigators traditionally pay attention to the relationship between cognitive processes and personality, the review of the existing literature uncovers a lack of research on the links between personality traits and short-term memory, as compared to the ample studies of the role which the working memory's central executive (Baddeley, 2003) plays regarding personality. In addition, these studies mostly examined particular traits or conventional personality taxonomies rather than their possible derivatives. In this regard, the present study addresses the role of reflexive characteristic adaptations as a structural element of personality system in terms of the five-factor theory (McCrae \& Costa, 1996, 2013). Spatial short-term memory, measured with Corsi test, correlated $(\mathrm{N}=1,030)$ with neuroticism and low openness to experience. On the contrary, verbal short-term memory, measured with the Sternberg task, correlated negatively with conscientiousness and agreeableness. Therefore, as compared to central executive, an increase in its slave subsystems spatial short-term memory and verbal short-term memory correlated with decreased agreeableness, conscientiousness, openness, and emotional stability.
\end{abstract}


Moreover, short-term memory demonstrated a range of links with reflexive characteristic adaptations including direct, indirect (mediated by traits), and compensatory (compared to traits) correlations. In particular, although neuroticism did not relate to verbal short-term memory, a negative attitude toward neuroticism did relate to this type of memory. In a similar fashion, although agreeableness did not relate to spatial short-term memory, a negative meta-attitude toward agreeableness (i.e., an attitude ascribed to one's parents) did relate to this type of memory. A negative link between conscientiousness and verbal short-term memory was supplemented with a positive attitude toward conscientiousness. The present findings are analysed in the context of previous studies, future implications and perspectives are discussed.

Keywords: five-factor theory; personality traits; characteristic adaptations; short-term memory; working memory.

\section{References}

Allik, J., \& Realo, A. (1997). Intelligence, academic abilities, and personality. Personality and Individual Differences, 23, 809-814. doi:10.1016/S0191-8869(97)00103-7

Avedisova, A. S., Fajzulloev, A. A., \& Bugaeva, T. P. (2004). Dinamika kognitivnykh funktsii u bol'nykh s emotsional'no-labil'nymi rasstroistvami sosudistogo geneza pri lechenii vazobralom [The dynamics of cognitive functions in patients with organic emotionally labile disorder of vascular genesis treated with Vasobral]. Klinicheskaya Farmakologiya i Terapiya, 13(2), 1-4.

Baddeley, A. (2003). Working memory: Looking back and looking forward. Nature Reviewos Neuroscience, 4, 829-839. doi:10.1038/nrn1201

Baddeley, A. D., \& Hitch, G. (1974). Working memory. In G. H. Bower (Ed.), Psychology of learning and motivation: Advances in research and theory (Vol. 8, pp. 47-89). New York: Academic Press.

Balabina, A. D. (2015). Refleksivnyie adaptatsii kharaktera i soziometricheskiy status [Reflexive characteristic adaptations and sociometric status]. Perm University Herald. Series "Philosophy. Psychology. Sociology", 2(22), 55-64.

Berkovits, I., Hancock, G. R., \& Nevitt, J. (2000). Bootstrap resampling approaches for repeated measure designs: Relative robustness to sphericity and normality violations. Educational and Psychological Measurement, 60, 877-892. doi:10.1177/00131640021970961

Bickel, W. K., Jarmolowicz, D. P., Mueller, E. T., Gatchalian, K. M., \& McClure, S. M. (2012). Are executive function and impulsivity antipodes? A conceptual reconstruction with special reference to addiction. Psychopharmacology, 221, 361-387. doi:10.1007/s00213-012-2689-x

Bishop, D. V. M., Adams, C. V., \& Norbury, C. F. (2006). Distinct genetic influences on grammar and phonological short-term memory deficits: evidence from 6-year-old twins. Genes, Brain and Behavior, 5, 158-169. doi:10.1111/j.1601-183X.2005.00148.x

Bull, R., Espy, K. A., \& Wiebe, S. A. (2008). Short-term memory, working memory, and executive functioning in preschoolers: Longitudinal predictors of mathematical achievement at age 7 years. Developmental Neuropsychology, 33, 205-228. doi:10.1080/87565640801982312

Campbell, A. M., Davalos, D. B., McCabe, D. P., \& Troup, L. J. (2011). Executive functions and extraversion. Personality and Individual Differences, 51, 720-725. doi:10.1016/j.paid.2011.06.018 
Compton, R. J. (2000). Ability to disengage attention predicts negative affect. Cognition and Emotion, 14, 401-415. doi:10.1080/026999300378897

Corsi, P. M. (1972). Human memory and the medial temporal region of the brain. Dissertation Abstracts International, 34(02), 891B. (University Microfilms No. AAI05-77717).

Costantini, G., Richetin, J., Borsboom, D., Fried, E. I., Rhemtulla, M., \& Perugini, M. (2015). Development of indirect measures of conscientiousness: Combining a facets approach and network analysis. European Journal of Personality, 29, 548-567. doi:10.1002/per.2014.

Cumming, G. (2014). The new statistics: Why and how. Psychological Science, 25, 7-29. doi:10.1177/0956797613504966

Derakshan, N., \& Eysenck, M. W. (2009). Anxiety, processing efficiency, and cognitive performance. European Psychologist, 14, 168-176. doi:10.1027/1016-9040.14.2.168

DeYoung, C. G. (2006). Higher-order factors of the Big Five in a multi-informant sample. Journal of Personality and Social Psychology, 91, 1138-1151. doi:10.1037/0022-3514.91.6.1138

DeYoung, C. G., Hirsh, J. B., Shane, M. S., Papademetris, X., Rajeevan, N., \& Gray, J. R. (2010). Testing predictions from personality neuroscience: Brain structure and the Big Five. Psychological Science, 21, 820-828. doi:10.1177/0956797610370159

DeYoung, C. G., Peterson, J. B., \& Higgins, D. M. (2005). Sources of openness/intellect: Cognitive and neuropsychological correlates of the fifth factor of personality. Journal of Personality, 73, 825-858. doi:10.1111/j.1467-6494.2005.00330.x

Eysenck, M., Payne, S., \& Derakshan, N. (2005). Trait anxiety, visuospatial processing, and working memory. Cognition and Emotion, 19, 1214-1228. doi:10.1080/02699930500260245

Ferini-Strambi, L., Baietto, C., Di Gioia, M. R., Castaldi, P., Castronovo, C., Zucconi, M., \& Cappa, S. F. (2003). Cognitive dysfunction in patients with obstructive sleep apnea (OSA): partial reversibility after continuous positive airway pressure (CPAP). Brain Research Bulletin, 61, 87-92. doi:10.1016/S0361-9230(03)00068-6

Feuerstein, C., Naлgele, B., Pepin, J.-L., \& Levy, P. (1997). Frontal lobe-related cognitive functions in patients with Sleep Apnea Syndrome before and after treatment: The relationship between sleep and cognitive functions during wakefulness. Acta Neurologica Belgica, 97, 96-107. Retrieved from http://cat.inist.fr/?aModele=afficheN\&cpsidt $=2714326$

Fleming, K. A., Heintzelman, S. J., \& Bartholow, B. D. (2015). Specifying associations between conscientiousness and executive functioning: Mental set shifting, not prepotent response inhibition or working memory updating. Journal of Personality. Advanced online publication. doi:10.1111/jopy.12163

Gathercole, S. E. (1999). Cognitive approaches to the development of short-term memory. Trends in Cognitive Sciences, 3, 410-419. doi:10.1016/S1364-6613(99)01388-1

Gray, J. R., \& Braver, T. S. (2002). Personality predicts working-memory-related activation in the caudal anterior cingulate cortex. Cognitive, Affective, and Behavioral Neuroscience, 2, 64-75. doi:10.3758/CABN.2.1.64

Hinson, J. M., Jameson, T. L., \& Whitney, P. (2003). Impulsive decision making and working memory. Journal of Experimental Psychology: Learning, Memory, and Cognition, 29, 298-306. doi:10.1037/0278-7393.29.2.298

Inquisit 3.0. (2015). Computer software. Seattle, WA: Millisecond Software.

Jarrold, C., \& Baddeley, A. D. (1997). Short-term memory for verbal and visuospatial information in Down’s syndrome. Cognitive Neuropsychiatry, 2, 101-122. doi:10.1080/135468097396351 
Jensen-Campbell, L. A., Rosselli, M., Workman, K. A., Santisi, M., Rios, J. D., \& Bojan, D. (2002). Agreeableness, conscientiousness, and effortful control processes. Journal of Research in Personality, 36, 476-489. doi:10.1016/S0092-6566(02)00004-1

John, O. P., Donahue, E. M., \& Kentle, R. L. (1991). The Big Five Inventory - Versions $4 a$ and 54. Berkeley, CA: University of California, Berkeley, Institute of Personality and Social Research.

John, O. P., Naumann, L. P., \& Soto, C. J. (2008). Paradigm shift to the integrative Big Five trait taxonomy: History, measurement, and conceptual issues. In O. P. John, R. W. Robins, \& L. A. Pervin (Eds.), Handbook of personality: Theory and research (pp. 114-158). New York: Guilford Press.

Lieberman, M. D., \& Rosenthal, R. (2001). Why introverts can't always tell who likes them: Multitasking and nonverbal decoding. Journal of Personality and Social Psychology, 80, 294-310. doi:10.1037/0022-3514.80.2.294

Linnenbrink, E. A., Ryan, A. M., \& Pintrich, P. R. (1999). The role of goals and affect in working memory functioning. Learning and Individual Differences, 11, 213-230. doi:10.1016/S10416080(00)80006-0

Malloy, T. E., Albright, L., Kenny, D. A., Agatstein, F., \& Winquist, L. (1997). Interpersonal perception and metaperception in nonoverlapping social groups. Journal of Personality and Social Psychology, 72, 390-398. doi:10.1037/0022-3514.72.2.390

McCrae, R. R., \& Costa, P. T., Jr. (1996). Toward a new generation of personality theories: Theoretical contexts for the five-factor model. In J. S. Wiggins (Ed.), The Five-factor model of personality: Theoretical perspectives (pp. 51-87). New York: Guilford Press.

McCrae, R. R., \& Costa, P. T., Jr. (2013). Introduction to the empirical and theoretical status of the five-factor model of personality traits. In T. A. Widiger \& P. T. Costa, Jr. (Eds.), Personality disorders and the five-factor model of personality (3rd ed., pp. 15-27). Washington, DC: American Psychological Association.

McLean, J. F., \& Hitch, G. J. (1999). Working memory impairments in children with specific arithmetic learning difficulties. Journal of Experimental Child Psychology, 74, 240-260. doi:10.1006/jecp.1999.2516

Moutafi, J., Furnham, A., \& Paltiel, L. (2004). Why is conscientiousness negatively correlated with intelligence? Personality and Individual Differences, 37, 1013-1022. doi:10.1016/ j.paid.2003.11.010

Murdock, K. W., Oddi, K. B., \& Bridgett, D. J. (2013). Cognitive correlates of personality: Links between executive functioning and the big five personality traits. Journal of Individual Differences, 34, 97-104. doi:10.1027/1614-0001/a000104

Muris, P., Bos, A. E. R., Mayer, B., Verkade, R., Thewissen, V., \& Dell'Avvento, V. (2009). Relations among behavioral inhibition, Big Five personality factors, and anxiety disorder symptoms in nonclinical children. Personality and Individual Differences, 46, 525-529. doi:10.1016/ j.paid.2008.12.003

Naegele, B., Pepin, J. L., Levy, P., Bonnet, C., Pellat, J., \& Feuerstein, C. (1998). Cognitive executive dysfunction in patients with obstructive sleep apnea syndrome (OSAS) after CPAP treatment. Sleep, 21, 392-397.

Nairne, J. S. (1996). Short-term/working memory. In E. L. Bjork \& R. A. Bjork (Eds.), Memory (pp. 101-128). San Diego, CA: Academic Press.

Park, S., \& McTigue, K. (1997). Working memory and the syndromes of schizotypal personality. Schizophrenia Research, 26, 213-220. doi:10.1016/S0920-9964(97)00051-0

Revelle, W. (2012). An introduction to psychometric theory with applications in R. Retrieved from http://www.personality-project.org/ 
Savine, A. C., McDaniel, M. A., Shelton, J. T., \& Scullin, M. K. (2012). A characterization of individual differences in prospective memory monitoring using the Complex Ongoing Serial Task.Journal of Experimental Psychology: General, 141, 337-362. doi:10.1037/a0025753

Semyashkin, A. A. (2010). Sootnoshenie kognitivnykh stilei i individual'no-psikhologicheskikh osobennostei lichnosti [The relationship between cognitive styles and individual characteristics of personality] (PhD dissertation, Sholokhov Moscow State University for Humanities, Moscow).

Shchebetenko, S. (2014). "The best man in the world": Attitudes toward personality traits. Psychology. Journal of the Higher School of Economics, 11(3), 129-148.

Shchebetenko, S. A. (2015a). Otrazhennye ustanovki na cherty lichnosti kak prediktor uspevaemosti studentov [Meta-attitudes toward personality traits as a predictor of academic achievement]. Psikhologiya i Psikhotekhnika, 1(76), 70-82. doi:10.7256/2070-8955.2015.1.13526

Shchebetenko, S. (2015b). Refleksivnye adaptatsii kharaktera v pyatifaktornoi teorii lichnosti [Reflexive characteristic adaptations within the five-factor theory of personality framework]. Psikhologicheskii Zhurnal, 36(6), 55-65.

Shchebetenko, S. A., \& Tutikova, E. A. (2015). "The picture is good because openness to experience is good": The mediating role of attitudes toward personality traits in individual differences in painting preferences. Psychology. Journal of Higher School of Economics, 12(4), 122-141. (in Russian)

Smith, E. E., \& Jonides, J. (1997). Working memory: A view from neuroimaging. Cognitive Psychology, 33, 5-42. doi:10.1006/cogp.1997.0658

Solso, R. (2006). Kognitionaya psikhologiya [Cognitive psychology]. Saint Petersburg: Piter. (Transl. of: Solso, R. (1995). Cognitive psychology (4th ed.). Boston: Allyn and Bacon).

Sternberg, S. (1966). High-speed scanning in human memory. Science, 153(3736), 652-654. doi:10.1126/science.153.3736.652

Unsworth, N., Heitz, R. P., Schrock, J. C., \& Engle, R. W. (2005). An automated version of the operation span task. Behavior Research Methods, 37, 498-505. doi:10.3758/BF03192720

Unsworth, N., Miller, J. D., Lakey, C. E., Young, D. L., Thadeus, J., Keith, W., \& Goodie, A. S. (2009). Exploring the relations among executive functions, fluid intelligence, and personality. Journal of Individual Differences, 30, 194-200. doi:10.1027/1614-0001.30.4.194

Wang, P. P., \& Bellugi, U. (1994). Evidence from two genetic syndromes for a dissociation between verbal and visual-spatial short-term memory. Journal of Clinical and Experimental Neuropsychology, 16, 317-322. doi:10.1080/01688639408402641

Wilcox, R., Carlson, M., Azen, S., \& Clark, F. (2013). Avoid lost discoveries, because of violations of standard assumptions, by using modern robust statistical methods. Journal of Clinical Epidemiology, 66, 319-329. doi:10.1016/j.jclinepi.2012.09.003

Williams, P. G., Suchy, Y., \& Kraybill, M. L. (2010). Five-Factor Model personality traits and executive functioning among older adults. Journal of Research in Personality, 44, 485-491. doi:10.1016/j.jrp.2010.06.002

Zaika, E. V., \& Kuznetsov, M. A. (1989). Kratkovremennaya pamyat' i usvoenie prakticheskikh umenii [Short-term memory and practical skills learning]. Voprosy Psikhologii, 2, 120-123.

Sergei A. Shchebetenko - senior research fellow, Department of Developmental Psychology, Perm State University, Ph.D.

E-mail: shebetenko@gmail.com 